\title{
Effects of Fuel Lewis Number on Localised Forced Ignition of Globally Stoichiometric Stratified Mixtures: a Numerical Investigation
}

\author{
Dipal Patel ${ }^{1} \cdot$ Nilanjan Chakraborty ${ }^{1}$
}

Received: 15 October 2015 / Accepted: 25 November 2015 / Published online: 13 February 2016

(C) The Author(s) 2016. This article is published with open access at Springerlink.com

\begin{abstract}
The influences of fuel Lewis number $L e_{F}$ on localised forced ignition of globally stoichiometric stratified mixtures have been analysed using three-dimensional compressible Direct Numerical Simulations (DNS) for cases with $L e_{F}$ ranging from 0.8 to 1.2. The globally stoichiometric stratified mixtures with different values of root-mean-square (rms) equivalence ratio fluctuation (i.e. $\phi^{\prime}=0.2,0.4$ and 0.6 ) and the Taylor micro-scale $l_{\phi}$ of equivalence ratio $\phi$ variation (i.e. $l_{\phi} / l_{f}=2.1,5.5$ and 8.3 with $l_{f}$ being the Zel'dovich flame thickness of the stoichiometric laminar premixed flame) have been considered for different initial rms values of turbulent velocity $u^{\prime}$. A pseudo-spectral method is used to initialise the equivalence ratio variation following a presumed bi-modal distribution for prescribed values of $\phi^{\prime}$ and $l_{\phi} / l_{f}$ for global mean equivalence ratio $\langle\phi\rangle=1.0$. The localised ignition is accounted for by a source term in the energy transport equation that deposits energy for a stipulated time interval. It has been observed that the maximum values of temperature and the fuel reaction rate magnitude increase with decreasing $L e_{F}$ during the period of external energy deposition. The initial values of $L e_{F}, u^{\prime} / S_{b(\phi=1)}, \phi^{\prime}$ and $l_{\phi} / l_{f}$ have been found to have significant effects on the extent of burning of the stratified mixtures following localised ignition. For a given value of $u^{\prime} / S_{b(\phi=1)}$, the extent of burning decreases with increasing $L e_{F}$. An increase in $u^{\prime}$ leads to a monotonic reduction in the burned gas mass for all values of $L e_{F}$ in all stratified mixture cases but an opposite trend is observed for the $L e_{F}=0.8$ homogeneous mixture. It has been found that an increase in $\phi^{\prime}$ has adverse effects on the burned gas mass, whereas the effects of $l_{\phi} / l_{f}$ on the extent of burning are non-monotonic and dependent on $\phi^{\prime}$ and $L e_{F}$. Detailed physical explanations have been provided for the observed $L e_{F}, u^{\prime} / S_{b(\phi=1)}, \phi^{\prime}$ and $l_{\phi} / l_{f}$ dependences.
\end{abstract}

Nilanjan Chakraborty

nilanjan.chakraborty@newcastle.ac.uk

Dipal Patel

d.patel2@ncl.ac.uk

1 School of Mechanical and Systems Engineering, Newcastle University, Newcastle Upon Tyne, NE1 7RU, UK 
Keywords Ignition - Stratified mixture $\cdot$ Equivalence ratio $\cdot$ rms turbulent velocity Direct Numerical Simulation

\section{Introduction}

Localised forced ignition of inhomogeneous mixtures plays a pivotal role in smooth functioning of Direct Injection (DI) engines and high altitude relight in aero-gas turbines and therefore improved understanding is needed to identify the conditions which lead to successful ignition and self-sustained combustion. Ignition of turbulent inhomogeneous fuel-air mixtures produced by evaporating droplets has been extensively analysed by Lefebvre [1] and Ballal and Lefebvre [2-4] from the viewpoints of critical spark energy, optimum spark duration and spark radius. Experimental data by Lefebvre [1] and Ballal and Lefebvre [24] suggests that an increase in root-mean-square (rms) value of turbulent velocity $u^{\prime}$ has adverse effects on successful ignition and early stages of combustion in the case of successful ignition. Similar adverse effects of $u^{\prime}$ have also been reported for homogeneous mixtures based on experimental $[1,5]$ and numerical $[6,7]$ analyses. Three-dimensional Direct Numerical Simulation (DNS) studies [8-13] also demonstrated that an increase in turbulent velocity fluctuation for a given value of integral length scale of turbulence is detrimental to the success of localised ignition of inhomogeneous gaseous and droplet-laden mixtures. The experimental data of Ahmed and Mastorakos [14] and Ahmed et al. [15] showed that an increase in mean velocity leads to a deterioration of ignition performance, quantified by a reduction in ignition probability. Most existing studies on localised forced ignition of inhomogeneous mixtures [8-10, 14, 15] have been carried out for a mixture distribution, which is characterised by a mean variation of equivalence ratio $\phi$. By contrast, relatively limited effort [16-18] has been directed to the ignition of stratified mixtures with a constant global mean equivalence ratio $\phi$ but for non-zero rms values of equivalence ratio fluctuations. Pera et al. [17] demonstrated that mixture inhomogeneity significantly affects flame wrinkling and cycle-to-cycle variations in Internal Combustion (IC) engines based on two-dimensional detailed chemistry based DNS simulations and a similar conclusion was previously drawn by Swaminathan et al. [16]. Recently, Patel and Chakraborty [18] used three-dimensional simple chemistry DNS simulations to demonstrate that the rms value of equivalence ratio fluctuation $\phi^{\prime}$ and the Taylor micro-scale $l_{\phi}$ of equivalence ratio $\phi$ variation, in addition to the rms values of turbulent velocity $u^{\prime}$, have significant effects on the extent of burning and the possibility of attaining self-sustained combustion following successful ignition.

A number of previous analyses [10, 19-22] have demonstrated that the Lewis number of the fuel, in addition to the mixture composition and background turbulence, has significant influences on localised forced ignition of both homogeneous and inhomogeneous gaseous mixtures. The Lewis number of species $i$ is defined as the ratio of its thermal diffusivity to mass diffusivity (i.e. $L e_{i}=\alpha_{i} / D_{i}$ ), and this non-dimensional number signifies the differential diffusion of heat and mass. Strehlow [19] and Glassman [20] demonstrated that the minimum ignition energy (MIE) for homogeneous mixtures is affected by the differential diffusion of heat and mass. The analytical study by Sibulkin and Siskind [21] indicated that the MIE for quiescent homogeneous mixtures decreases with increasing $D_{i}$ for a given value of thermal diffusivity (i.e. with decreasing Lewis number). Furthermore, He [22] analytically demonstrated that localised ignition of quiescent homogeneous mixtures with fuel Lewis number $L e_{F}>1.0$ can be unstable. This is indeed demonstrated by 
Chakraborty et al. [10] that it is easier to ignite mixing layers and obtain self-sustained combustion for small values of fuel Lewis number and this tendency is particularly prevalent for cases with $L e_{F}<1.0$. From the above discussion, it becomes evident that $L e_{F}$ is also likely to play key roles on the localised forced ignition of stratified mixtures but these effects are yet to be analysed in detail using DNS. This deficit in the existing literature has been addressed in the current study by carrying out three-dimensional compressible DNS for a range of different values of $L e_{F}, u^{\prime}, \phi^{\prime}$ and $l_{\phi}$. The Taylor micro-scale of the equivalence ratio variation $l_{\phi}$ is defined as [23]:

$$
l_{\phi}=\sqrt{\frac{6\left\langle[\phi-\langle\phi\rangle]^{2}\right\rangle}{\langle\nabla[\phi-\langle\phi\rangle] \cdot \nabla[\phi-\langle\phi\rangle]\rangle}}
$$

where the angled bracket indicates the global mean evaluated over the whole of computational domain. In the present study, globally stoichiometric (i.e. $\langle\phi\rangle=1.0$ ) stratified mixtures have been considered for different values of $\phi^{\prime}$ and $l_{\phi}$. For the present analysis, the equivalence ratio $\phi$ variation is initialised using a presumed bi-modal distribution for prescribed values of $\phi^{\prime}$ and $l_{\phi}$ for $\langle\phi\rangle=1.0$ using a methodology proposed by Eswaran and Pope [23]. This methodology for the initialisation of equivalence ratio was used for DNS of stratified mixture combustion in a number of previous DNS studies [16-18, 24-26]. The main objectives of the present study are:

- To understand the influences of fuel Lewis number $L e_{F}$ on the possibility of obtaining successful ignition and sustainability of the subsequent combustion

- To demonstrate the effects of $u^{\prime}, \phi^{\prime}$ and $l_{\phi}$ on localised forced ignition of stratified mixtures and early stages of combustion in the case of successful ignition

- To provide physical explanations for the observed $L e_{F}, u^{\prime}, \phi^{\prime}$ and $l_{\phi}$ dependences of the extent of burning following successful ignition of stratified mixtures

The rest of the paper is organised as follows. The mathematical background and numerical implementation relevant to the current analysis will be presented in the next section. Following this, results will be presented and subsequently discussed. The main findings will be summarised and conclusions will be drawn in the final section of this paper.

\section{Mathematical Background and Numerical Implementation}

Three-dimensional detailed chemistry DNS simulations still remain extremely expensive [27] for a detailed parametric analysis, as carried out in this analysis. Thus, the chemical mechanism is simplified here by a modified single-step chemical reaction [28]:

$$
\text { Fuel }+s \cdot \text { Oxidiser } \rightarrow(1+s) \cdot \text { Products }
$$

where $s$ indicates the mass of oxidiser consumed per unit mass of fuel consumption under stoichiometric conditions. The fuel reaction rate is given by an Arrhenius type expression:

$$
\dot{w}_{F}=-\rho B^{*} Y_{F} Y_{O} \exp \left[\frac{-\beta(1-T)}{1-\alpha(1-T)}\right]
$$


where $Y_{F}$ and $Y_{O}$ are the local fuel and oxidiser mass fractions respectively, $\rho$ is the gas density, $\beta$ is the Zel'dovich number, $\alpha$ is the heat release parameter, $B^{*}$ is a normalized pre-exponential factor and $T$ is the non-dimensional temperature which are defined as:

$$
\begin{gathered}
\beta=\frac{E_{a}\left(T_{a d(\phi=1)}-T_{0}\right)}{R T_{a d(\phi=1)}^{2}} ; \alpha=\frac{\tau}{1+\tau}=\frac{T_{a d(\phi=1)}-T_{0}}{T_{a d(\phi=1)}} \\
B^{*}=B \exp \left(-\frac{\beta}{\alpha}\right) ; T=\frac{\hat{T}-T_{0}}{T_{a d(\phi=1)}-T_{0}}
\end{gathered}
$$

where $E_{a}$ is the activation energy, $R$ is the gas constant, $B$ is the pre-exponential factor, $\tau=\left(T_{a d(\phi=1)}-T_{0}\right) / T_{0}$ is the heat release parameter, $\hat{T}$ is the instantaneous dimensional temperature, $T_{0}$ is the initial reactant temperature and $T_{a d(\phi=1)}$ is the adiabatic flame temperature for the stoichiometric mixtures. Here the Zel'dovich number $\beta$ and the adiabatic flame temperature $T_{a d(\phi=1)}$ are taken to be functions of equivalence ratio $\phi$ following Tarrazo et al. [28] so that the modified one-step chemistry captures the realistic $\phi$ dependence of unstrained laminar burning velocity $S_{b(\phi)}$. The Zel'dovich number $\beta$ is expressed as $\beta=6 f(\phi)$ according to Tarrazo et al. [28] where $f(\phi)$ is given by:

$$
f(\phi)=\left\{\begin{array}{llcrl}
1+8.25(\phi-1)^{2} & \text { for } & \phi & \leq 0.64 \\
1+1.443(\phi-1.07)^{2} & \text { for } & \phi & \geq 1.07 \\
1 & \text { for } & 0.64 & <\phi<1.07
\end{array}\right.
$$

The heat release per unit mass of fuel $H_{\phi}$ is expressed according to Tarrazo et al. [28] in the following manner: $H_{\phi} / H_{\phi=1}=1$ for $\phi \leq 1$ and $H_{\phi} / H_{\phi=1}=1-\alpha_{H}(\phi-1)$ for $\phi>1$, where $H_{\phi=1}=\left[\left(T_{a d(\phi=1)}-T_{0}\right) C_{P}\right] / Y_{F 0(\phi=1)} \quad \alpha_{H}=0.21$ and $Y_{F 0(\phi=1)}$ is the fuel mass fraction in the unburned gas for a premixed flame of equivalence ratio $\phi=1.0$.

The combustion is assumed to be taking place in the gaseous phase where all species are considered to be perfect gases. Standard values are taken for the ratio of specific heats $\left(\gamma=C_{P} / C_{V}=1.4\right)$ and Prandtl number $\left(\operatorname{Pr}=\mu C_{P} / \lambda=0.7\right)$. Here the heat release parameter $\tau$ is taken to be 3.0 for all cases (i.e. $\tau=3.0$ ) and thermo-physical properties such as viscosity $\mu$, thermal conductivity $\lambda$ and density-weighted mass diffusivity $\rho D$ are taken to be constant and independent of temperature following several previous analyses [12, 13, 29-32].

Mixture inhomogeneity in stratified mixtures is often quantified in terms of mixture fraction $\xi$, which can be expressed in terms of both fuel and oxidiser mass fractions (i.e. $Y_{F}$ and $\left.Y_{O}\right)$ as [33]:

$$
\xi=\frac{\left(Y_{F}-Y_{O} / s+Y_{O \infty} / s\right)}{\left(Y_{F \infty}+Y_{O \infty} / s\right)}
$$

where $Y_{F \infty}$ is the fuel mass fraction in the pure fuel stream and $Y_{O \infty}$ is the oxidiser mass fraction in air. The equivalence ratio $\phi$ can be expressed in terms of $\xi$ and the stoichiometric mixture fraction $\xi_{s t}$ as:

$$
\phi=\frac{\left(1-\xi_{s t}\right) \xi}{(1-\xi) \xi_{s t}} \quad \text { where } \quad \xi_{s t}=\frac{Y_{O \infty}}{s Y_{F \infty}+Y_{O \infty}}
$$

For the present analysis $s=4 ; Y_{F \infty}=1.0$ and $Y_{O \infty}=0.233$ have been taken which represent methane-air binary mixture. These values lead to $Y_{F s t}=0.055$ and $\xi_{s t}=0.055$. The extent of the completion of the chemical reaction for inhomogeneous mixtures can be expressed in terms of a reaction progress variable $c$, which is defined as $[8-10,18,24]$ :

$$
c=\frac{\xi Y_{F \infty}-Y_{F}}{\xi Y_{F \infty}-\max \left[0, \frac{\xi-\xi_{s t}}{1-\xi_{s t}}\right] Y_{F \infty}}
$$


According to Eq. 8, $c$ rises monotonically from 0 in the fully unburned reactants to 1.0 in the fully burned products.

The localised ignition is modelled by heat addition due to a source term $q^{\prime \prime \prime}$ in the energy transport equation as done in several previous analyses $[8-13,18]$. The source term $q^{\prime \prime \prime}$ is assumed to follow a Gaussian distribution in the radial direction away from the centre of the ignitor [8-13, 18, 34, 35] and is expressed in the following manner:

$$
q^{\prime \prime \prime}(r)=A_{q} \exp \left(-\frac{r^{2}}{2 R^{2}}\right)
$$

where $r$ is the radial direction from the centre of the ignitor and $R$ is the width of the Gaussian profile, which is taken to be $R=1.10 l_{f}$ where $l_{f}=\alpha_{T} / S_{b(\phi=1)}$ is the Zel'dovich flame thickness with $\alpha_{T}$ and $S_{b(\phi=1)}$ being the unburned gas thermal diffusivity and unstrained laminar burning velocity of the stoichiometric fuel-air mixture respectively. This choice allows for the sufficient resolution of the temperature gradient and guarantees the rapid disappearance of any artificial effects introduces by the ignition source $[12,13,32$, 36]. The constant $A_{q}$ in Eq. 10 is determined by the volumetric integration $\dot{Q}=\int_{V} q^{\prime \prime \prime} d V$, where $\dot{Q}$ is the ignition power, which is defined as:

$$
\dot{Q}=a_{s p} \rho_{0} C_{P} \tau T_{0}\left(\frac{4}{3} \pi l_{f}^{3}\right)\left[\frac{H_{1}(t)-H_{2}\left(t-t_{s p}\right)}{t_{s p}}\right]
$$

where $a_{s p}$ is a parameter that determines the total energy deposited by the ignitor and here $a_{s p}$ is taken to be 3.6 following previous analyses [8-13, 18]. In Eq. 10, $t_{s p}=b_{s p} t_{f}$ is the energy deposition duration where $b_{s p}$ is the energy deposition duration parameter and $t_{f}$ is the characteristic chemical time scale (i.e. Zel'dovich time scale) given by $t_{f}=l_{f} / S_{b(\phi=1)}$. The parameter $b_{s p}$ for optimum spark duration varies between 0.2 and 0.4 [37], and $b_{s p}=$ 0.2 has been considered for the current investigation following several previous analyses [8$13,18,36]$. The details of spark formation (momentum modification contribution, plasma formation and shock wave) are kept beyond the scope of the present analysis in order to keep this study computationally feasible. An increase in $a_{s p}$ increases the ignition energy input and thus improves the chances of successful ignition, self-sustained combustion in the case of successful ignition, and increases the extent of burning (e.g. burned gas mass). By contrast, an increase in $b_{s p}$ for a given set of values of $R$ and $a_{s p}$ reduces ignition power and thus has detrimental effects on the chances of successful ignition, self-sustained combustion and extent of burning. Moreover, an increase in $R$ for a given set of values of $a_{s p}$ and $b_{s p}$ leads to deposition of same amount of ignition energy over a large volume (and mass) of gas and thus reduces the probability of finding large values of temperature, which in turn has detrimental effects on the success of ignition and subsequent sustenance of the flame. The effects of $R, a_{s p}$ and $b_{s p}$ on localised forced ignition for homogeneous mixtures have been addressed elsewhere [38] and similar qualitative effects can also be expected for stratified mixture combustion.

For the present analysis, the initial $\phi$ variation is specified by a random bi-modal distribution following a pseudo-spectral method proposed by Eswaran and Pope [23]. This method was used for initializing the mixture stratification in a number of previous analyses [24-26]. For a system, where fuel is injected in the form of the droplets, the evaporation sites are likely to be fuel-rich, whereas fuel-lean mixtures are likely to be encountered in the locations far away from droplets. This situation can be idealized by a bi-modal distribution of equivalence ratio $\phi$. For the current analysis, a globally stoichiometric (i.e. $\langle\phi\rangle=1.0$ ) mixture has been considered. The fuel Lewis number $L e_{F}$, initial values of the normalised 
turbulent velocity fluctuation $u^{\prime} / S_{b(\phi=1)}$ (where the ratio of longitudinal integral length scale to flame thickness $L_{11} / l_{f}=3.36$ for all cases), rms of equivalence ratio $\phi^{\prime}$ and normalised Taylor micro-scale of equivalence ratio variation $l_{\phi} / l_{f}$ are listed in Table 1 . For all cases the Lewis number of the oxidiser is taken to be unity (i.e. $L e_{O}=1.0$ ). A typical realisation for initial equivalence ratio $\phi$ distribution for initial values of $l_{\phi} / l_{f}=2.1,5.5$ and 8.3 is shown in Fig. 1 for initial $\phi^{\prime}=0.2$.

A compressible three-dimensional DNS code SENGA [39] was used to carry out the simulations. The simulation domain is taken to be a cube of size $33 l_{f} \times 33 l_{f} \times 33 l_{f}$, which ensures that about 10 integral eddies are retained on each side of the domain (i.e. longitudinal integral length scale $L_{11}=3.36 l_{f}$ ). The simulation domain is discretised by a Cartesian grid of size $200 \times 200 \times 200$ with uniform grid spacing ensuring 10 grid points within the thermal flame thickness $\delta_{t h(\phi=1)}=\left[T_{a d(\phi=1)}-T_{0}\right] / \operatorname{Max}|\nabla \hat{T}|_{L}$ of the fuel-air stoichiometric mixture, which ensures $\eta>\Delta x$ where $\eta$ is the Kolmogorov length scale. Four different realisations of 27 variations of $\phi$ distribution for each set of values of $u^{\prime}$ and $L e_{F}$ have been considered here, which amounts to altogether 333 simulations $(=27 \times 4 \times 3=324$ stratified mixtures cases +9 homogeneous mixture cases). The boundaries in the $x_{1}$ - direction are taken to be partially non-reflecting and are specified using the Navier-Stokes Characteristic Boundary Conditions formulation [40], whereas the boundaries in the other directions are considered to be periodic. High order finite-difference and Runge-Kutta schemes are used for spatial discretization and explicit time advancement respectively [39]. The turbulent velocity fluctuations are initialised by an incompressible homogeneous isotropic field, which is generated using a standard pseudo-spectral method [41]. Simulations under decaying turbulence should be carried out for a time $t_{\text {sim }} \geq \operatorname{Max}\left(t_{e}, t_{f}\right)$, where $L_{11} / \sqrt{k}_{0}$ is the initial eddy turn over time where $k_{0}$ is the initial turbulent kinetic energy evaluated over the whole domain. Statistics for all cases are presented at $t=8.40 t_{s p}=1.68 t_{f}$, which corresponds to about $2 t_{e}$ and $3 t_{e}$ for initial values of $u^{\prime} / S_{b(\phi=1)}=4.0$ and $u^{\prime} / S_{b(\phi=1)}=6.0$ respectively, and these simulation times remain either comparable to or greater than the simulation duration in several previous analyses on stratified mixture combustion and localised forced ignition in existing literature [12, 13, 16-18, 32, 36, 42-44].

\section{Results and Discussion}

\subsection{Global behaviour of the non-dimensional temperature field}

The temporal evolutions of the maximum values of non-dimensional temperature (i.e. $T_{\max }=\left(\hat{T}_{\max }-T_{0}\right)\left(T_{a d(\phi=1)}-T_{0}\right)$ are shown in Figs. 2 and 3 for initial values of $\phi^{\prime}=0.2$, and 0.6 cases respectively. The cases with initial $\phi^{\prime}=0.4$ are not explicitly shown here because the temporal evolution of $T_{\max }$ for these cases are mostly qualitatively similar to that of the initial $\phi^{\prime}=0.6$ cases except for the quiescent $L e_{F}=1.0$ and 1.2 cases with initial $l_{\phi} / l_{f}=5.5$, which exhibit self-sustained combustion for initial $\phi^{\prime}=0.4$ whereas the flames extinguish after successful ignition for initial $\phi^{\prime}=0.6$.

It can be seen from Figs. 2 and 3 that $T_{\max }$ rises with time due to energy deposition during $0 \leq t \leq t_{s p}$ and thermal runaway takes place once $T_{\max }$ attains a value close to $T_{c} \approx 1-\left(1 / \beta_{\phi=1}\right)$ leading to rapid increase in $T_{\max }$ with time until $t=1.0 t_{s p}$. The high thermal gradient between hot gas and surrounding unburned gas gives rise to high rate of heat transfer from the ignition kernel. This, in turn, leads to a decrease in $T_{\max }$ with time once the energy deposition is switched off but, $T_{\max }$ eventually settles to the 


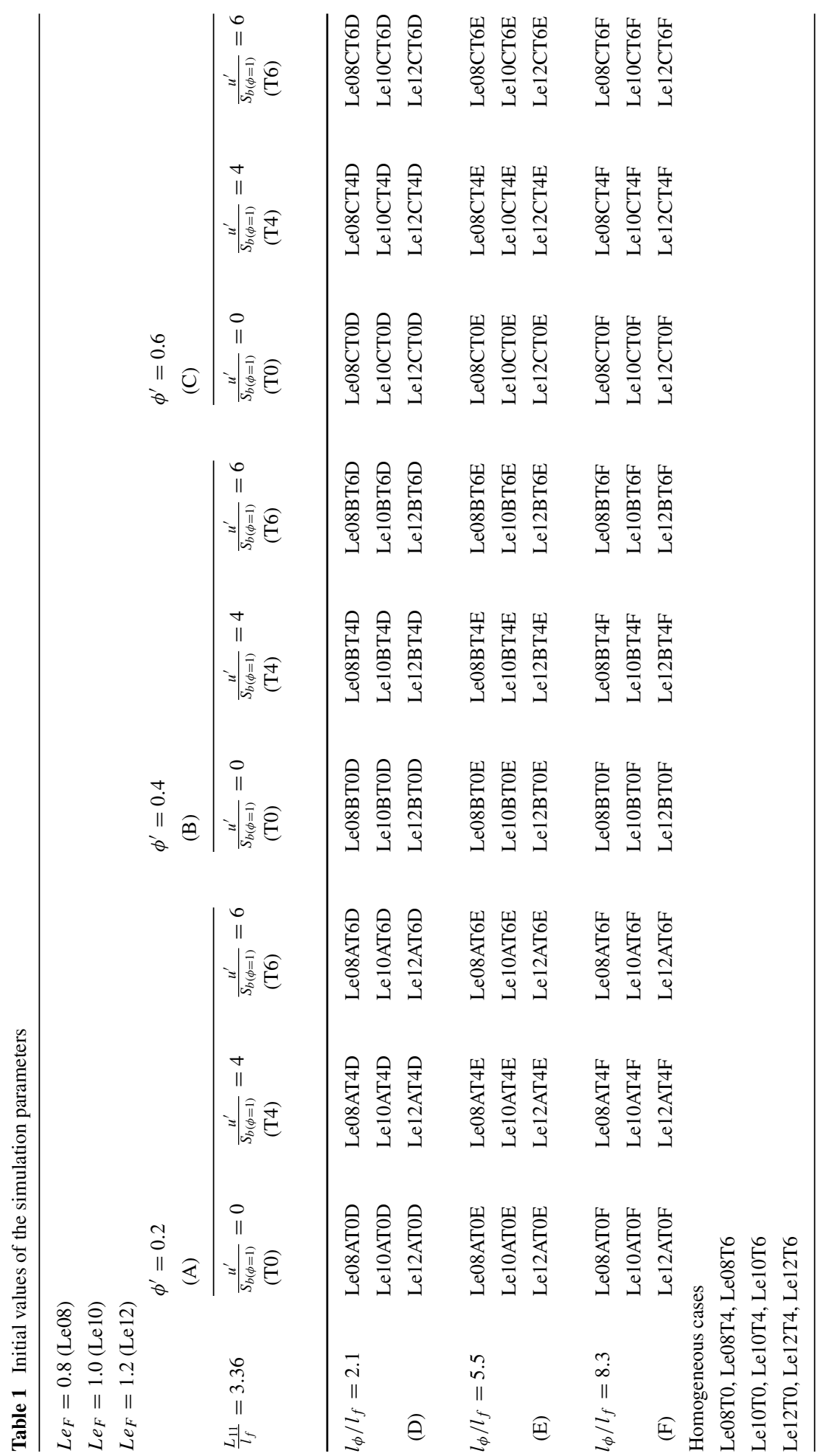



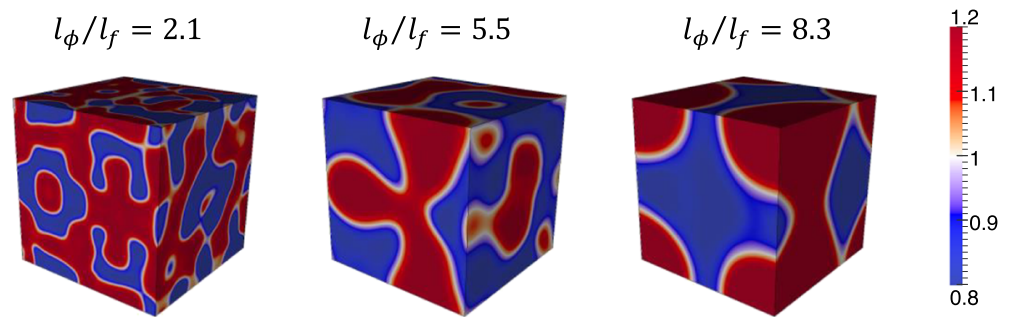

Fig. 1 A typical realisation of initial equivalence ratio $\phi$ distribution for $l_{\phi} / l_{f}=2.1,5.5$ and 8.3 corresponding to $\phi^{\prime}=0.2$. The domain size is $33 l_{f} \times 33 l_{f} \times 33 l_{f}$

non-dimensional adiabatic flame temperature of the stoichiometric mixtures (i.e. $T \approx 1.0$ ) in the cases where self-sustained combustion is obtained following successful ignition. It can be seen from Figs. 2 and 3 that self-sustained combustion has been obtained for all cases with $L e_{F}=0.8$, whereas only some cases with $L e_{F}=1.0$ and 1.2 exhibit self-sustained combustion. The conditions, which led to self-sustained flame propagation following successful ignition, depend on $L e_{F}, l_{\phi} / l_{f}, \phi^{\prime}$ and $u^{\prime} / S_{b(\phi=1)}$. It has been found that self-sustained flame propagation has been obtained for all the quiescent cases except for the Le10CT0E, Le12BT0E, Le12CT0D and Le12CT0E cases.

The maximum temperature value $T_{\max }$ decreases continuously with time for $t>t_{s p}$ in all the turbulent cases with $L e_{F}=1.2$ considered here, indicating failure to obtain selfsustained combustion in these cases and flame extinguishes at an earlier time for higher values of $u^{\prime}$. This is consistent with analytical results by He [22] who analytically demonstrated that it is more difficult to ignite and sustain combustion for $L e_{F}>1$. Moreover Figs. 2 and 3 demonstrate that $T_{\max }$ decreases continuously with time for $t>t_{s p}$ in the turbulent cases with $L e_{F}=1.0$ for initial values of $l_{\phi} / l_{f}=5.5$, and $\phi^{\prime}=0.6$, whereas selfsustained combustion is obtained for all the turbulent cases with initial values of $l_{\phi} / l_{f}=5.5$ for $L e_{F}=0.8$. The observations made from Figs. 2 and 3 indicate that $L e_{F}, l_{\phi} / l_{f}, \phi^{\prime}$ and $u^{\prime} / S_{b(\phi=1)}$ have important influences on the possibility of self-sustained combustion following successful ignition. Moreover, the effects of $l_{\phi} / l_{f}$ on the success of self-sustained combustion seem to be non-monotonic (e.g. the cases with initial $l_{\phi} / l_{f}=5.5$ are more prone to flame extinction than the cases with initial values of $l_{\phi} / l_{f}=2.1$ and 8.3 for $\left.\phi^{\prime}=0.6\right)$ in the case of $L e_{F}=1.0$.

\subsection{Spatial distributions of fuel mass fraction, temperature, fuel reaction rate and equivalence ratio}

The distributions of fuel mass fraction (i.e. $Y_{F}$ ), non-dimensional temperature (i.e. $T$ ), normalised fuel reaction rate magnitude (i.e. $\dot{\Omega}_{F}=\left|\dot{w}_{F}\right| \times l_{f} / \rho_{0} S_{b(\phi=1)}$ ) and equivalence ratio (i.e. $\phi$ ) at $t=1.05 t_{s p}$ and $t=8.40 t_{s p}$ in the central $x_{1}-x_{2}$ plane for the cases Le08BT4F, Le10BT4F and Le12BT4F are shown in Fig. 4. Similar qualitative behaviour has been observed for other cases but the burned gas volume for the stratified mixture cases has been found to decrease with increasing $u^{\prime} / S_{b(\phi=1)}$ for a given set of values of $L e_{F}, \phi^{\prime}$ and $l_{\phi} / l_{f}$.

It can be seen from Fig. 4 that the contours of $T$ remain approximately spherical during the period of energy deposition but they become increasingly wrinkled as time progresses for all turbulent cases. The evolution of $T$ is principally determined by the diffusion of deposited energy during the energy deposition period (i.e. $0<t<t_{s p}$ ), whereas after ignition, the evolution of isotherms depends on the magnitude of the reaction rate at the local mixture composition and the stretch rate induced by the background fluid motion. The local 

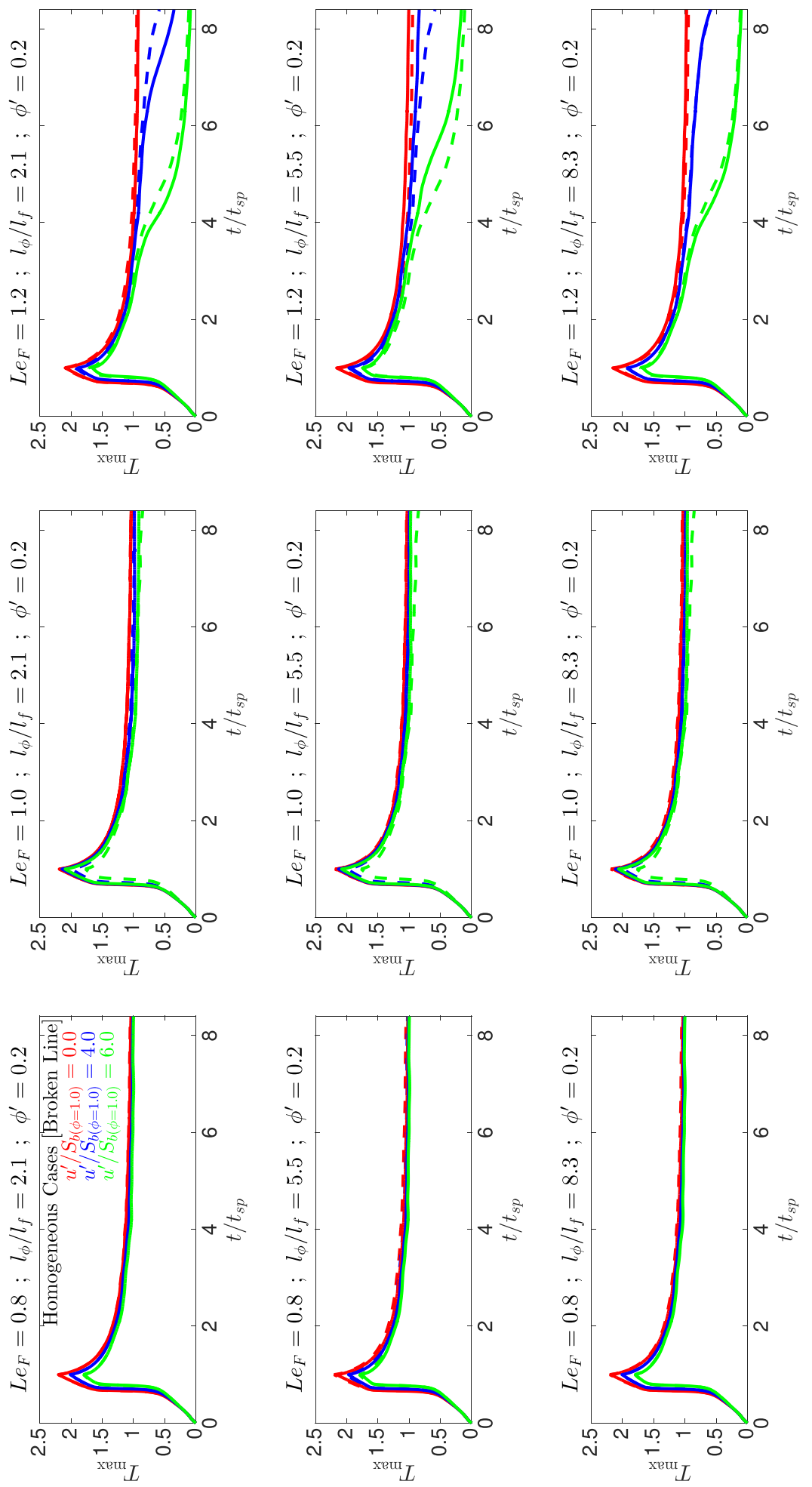

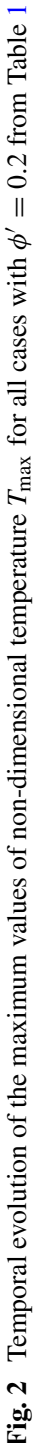



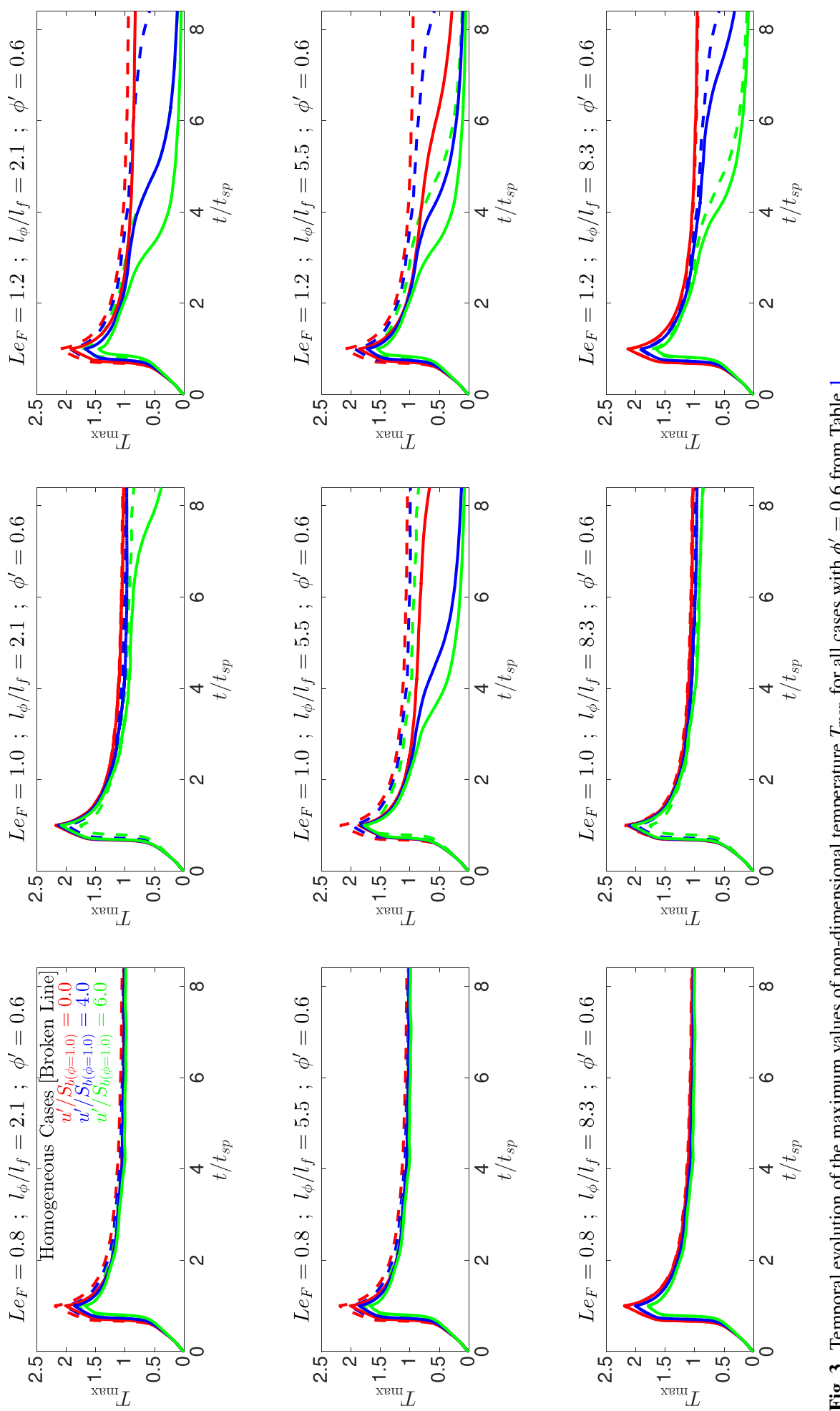

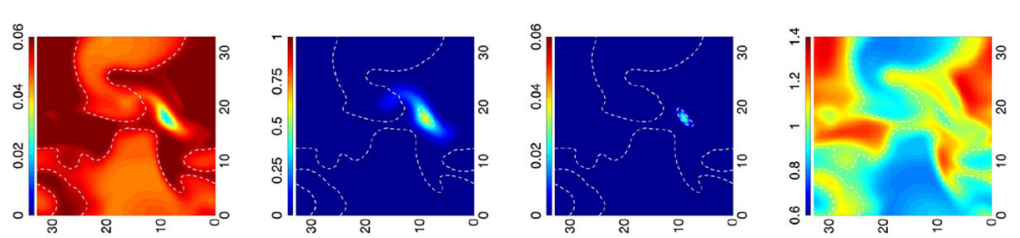

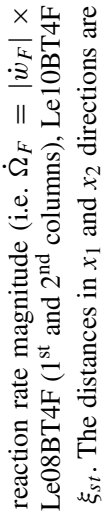
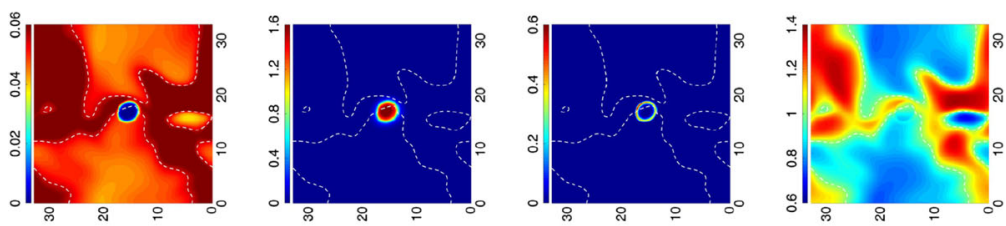

)

ఫัँ

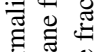

吾要

î̀

○ा

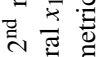
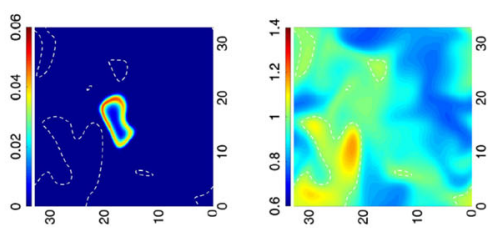

1 苞

$\stackrel{\circ}{\oplus} \stackrel{0}{\Xi}$

$\because \cong$

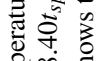

过

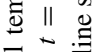

클 छ

क्ष

ฮั

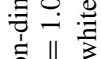

更

䒕

วิ

क छ

1 ठ

$\lambda \theta$

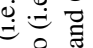

을

氙 突

कै तै तै

号

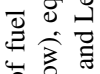

ㅎํํ छ

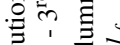

흘 응

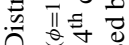

+ 桴产

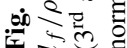


flame propagation statistics in response to flame stretch rate for the cases with successful combustion are found to be qualitatively similar to those discussed by Malkeson and Chakraborty [45] and thus are not repeated here. It can be seen from Fig. 4 that the level of non-uniformity in $\phi$ distribution decreases as time progresses in all cases. A comparison between different $L e_{F}$ cases reveals that the volume of high temperature region and the extent of flame wrinkling decrease with increasing fuel Lewis number. For the cases with $L e_{F}<1$, fuel diffuses faster into the reaction zone than the rate at which heat is conducted out, whereas the opposite mechanism remains prevalent for the $L e_{F}>1$ cases. As a result of this, simultaneous presence of high (low) concentration of fuel and high (low) temperature leads to higher (smaller) rate of burning and greater extent of flame wrinkling in the $L e_{F}<1\left(L e_{F}>1\right)$ cases than in the corresponding $L e_{F}=1$ cases.

\subsection{Mode of combustion and mixing statistics}

It is important to understand the flame structure originating from localised ignition to explain the observed burning behaviour of the cases considered here. The mode of combustion can be characterised by the flame index $I_{c}=\nabla Y_{F} \cdot \nabla Y_{O} /\left(\left|\nabla Y_{F}\right|\left|\nabla Y_{O}\right|\right)$, which assumes positive (negative) values for premixed (non-premixed) mode of combustion [24, 46]. The percentage of overall heat release arising from premixed (i.e. $I_{c}>0$ ) mode of combustion for the selected cases at $t=8.40 t_{s p}$ is shown in Table 2. It is evident from Table 2 that the chemical reaction takes place predominantly in premixed mode but some pockets of non-premixed combustion are also found in these cases. However, the probability of finding $I_{c}<0$ decreases with increasing time due to mixing process (not shown here).

Table 2 Percentage of heat release arising from premixed $\left(I_{c}>0\right)$ mode of combustion at $t=8.40 t_{s p}$ for selected cases where $I_{c}=\nabla Y_{F} \cdot \nabla Y_{O} /\left(\left|\nabla Y_{F}\right|\left|\nabla Y_{O}\right|\right)$ is the flame index

Case

$\%$ of heat release due to premixed mode of combustion

(a) Effects of $L e_{F}$

Le08AT6F

99.40

Le10AT6F

95.20

Le12AT6F

87.50

(b) Effects of $\phi^{\prime}$

Le10AT6F

95.20

Le10BT6F

89.60

Le10CT6F

83.70

(c) Effects of $l_{\phi} / l_{f}$

Le10AT4D

99.00

Le10AT4E

96.50

Le10AT4F

95.00

(d) Effects of $u^{\prime} / S_{b(\phi=1.0)}$

Le10BT0F

79.80

Le10BT4F

82.40

Le10BT6F

89.60 
It can be seen from Table 2 that the percentage of heat release from the premixed (i.e. $\left.I_{c}>0\right)$ mode of combustion decreases with increasing $L e_{F}$. Higher fuel diffusivity for small values of $L e_{F}$ augments the mixing rate and thus strengthens the contribution of premixed combustion to overall heat release. Moreover, the proportion of heat release originating from non-premixed combustion (i.e. $I_{c}<0$ ) increases with increasing $\phi^{\prime}$ for a given value of $l_{\phi} / l_{f}$ because the extent of non-premixed combustion is expected to increase with increasing extent of mixture inhomogeneity (see Table 2). Furthermore, the percentage of heat release arising from non-premixed mode of combustion decreases with decreasing (increasing) values of $l_{\phi} / l_{f}\left(u^{\prime} / S_{b(\phi=1)}\right)$ for a given value of $\phi^{\prime}$ as a result of improved mixing for small (high) values of $l_{\phi} / l_{f}\left(u^{\prime} / S_{b(\phi=1)}\right)$ because the mean scalar dissipation rate of mixture fraction $<N_{\xi}>=<D_{\xi} \nabla \xi \cdot \nabla \xi>$ scales as $<N_{\xi}>\sim D_{\xi}<\xi^{\prime \prime} \xi^{\prime \prime}>/ l_{\phi}^{2}$ [18] where $<\xi^{\prime \prime} \xi^{\prime \prime}>$ is the variance of mixture fraction. It has been demonstrated in Ref. [18] that a decrease (an increase) in $l_{\phi} / l_{f}\left(u^{\prime} / S_{b(\phi=1)}\right)$ augments the rate of mixing for $L e_{F}=1.0$ cases and thus is not repeated here because the same qualitative behaviour has been observed for $L e_{F}=0.8$ and 1.2 .

The evolution of mixing process can be illustrated by the temporal evolution of the pdfs of $\phi$, which is shown in Fig. 5a-d for the selected cases in order to demonstrate the effects of $L e_{F}$ and initial values of $l_{\phi} / l_{f}, \phi^{\prime}$ and $u^{\prime} / S_{b(\phi=1)}$. The temporal evolution of the rms value of equivalence ratio evaluated over the whole domain $\phi^{\prime}$ for the corresponding cases are shown in Fig. 5e-h. Figure 5a-d show that initial bi-modal distribution of $\phi$ approaches an approximate Gaussian distribution with peak value at $\phi \approx\langle\phi\rangle=1.0$, as time progresses. It can be seen from Figs. 5e-h that the decay rate of $\phi^{\prime}$ increases with increasing $u^{\prime} / S_{b(\phi=1)}$ as turbulent straining acts to increase scalar dissipation rate [47] which in turn increases the greater rate of micro-mixing. It can be seen from Fig. 5 that the decay rate of $\phi^{\prime}$ is stronger for decreasing (increasing) values of $L e_{F}$, and $l_{\phi} / l_{f}\left(u^{\prime} / S_{b(\phi=1)}\right)[10,18]$.

\subsection{Structure of the reaction zone and statistical behaviour of fuel reaction rate magnitude}

It is important to understand the reaction zone structure of the flames initiated by the localised forced ignition, in order to explain the effects of stratification on the extent of burning following successful ignition. The scatter of $\dot{\Omega}_{F}=\left|\dot{w}_{F}\right| \times l_{f} / \rho_{0} S_{b(\phi=1)}$ with reaction progress variable $c$ is presented in Fig. 6 ( $1^{\text {st }}$ row) at $t=8.40 t_{s p}$ for the cases Le08BT4F, Le10BT4F, Le12BT4F and a similar qualitative behaviour has been observed for all cases where self-sustained combustion is obtained.

Figure 6 ( $1^{\text {st }}$ row) shows that the high values of $\dot{\Omega}_{F}$ are obtained close to $c=0.8$, which is consistent with previous analyses [8-10, 18]. The scatter of $\dot{\Omega}_{F}$ with mixture fraction $\xi$ for the cases Le08BT4F, Le10BT4F, Le12BT4F is presented in Fig. 6 ( $2^{\text {nd }}$ row), which shows that a considerable amount of scatter, and the same qualitative behaviour is observed for other cases. The large variation of non-dimensional temperature $T$ on a given $\xi$ isosurface (due to both unburned and burned contributions) leads to a large extent of scatter of $\dot{\Omega}_{F}$. Furthermore Fig. 6 ( $2^{\text {nd }}$ row) shows that the high values of $\dot{\Omega}_{F}$ are obtained towards the slightly rich side (i.e. $\xi \approx 0.06$ ) which corresponds to $\phi \approx 1.10$, where the unstrained planar laminar burning velocity attains its maximum value.

The temporal evolution of $\dot{\Omega}_{F}$ conditional of mixture fraction $\xi$ in the region corresponding to $0.01 \leq c \leq 0.99$ for the cases Le08BT4F, Le10BT4F, Le12BT4F is shown in Fig. 6 ( $3^{\text {rd }}$ row) for different time instants. It can be seen from Fig. 6 that the maximum values of $\dot{\Omega}_{F}$ decreases with increasing $L e_{F}$ (e.g. the maximum value of $\dot{\Omega}_{F}$ is highest for the $L e_{F}=0.8$ case and the lowest in the $L e_{F}=1.2$ case). In the $L e_{F}<1$ case, fuel diffuses 


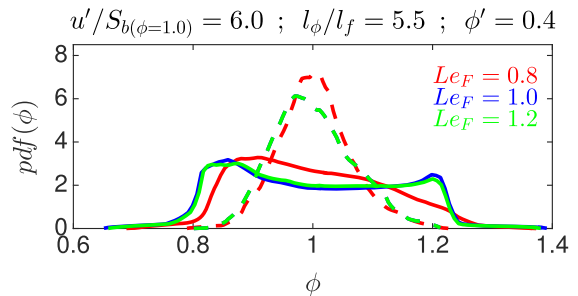

(a)

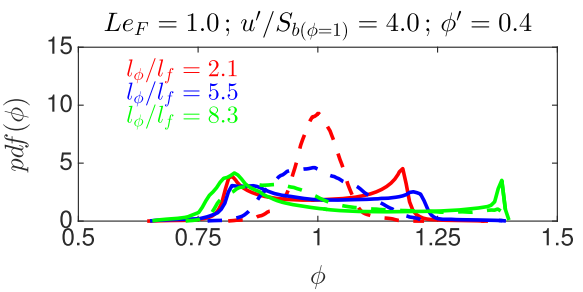

(c)

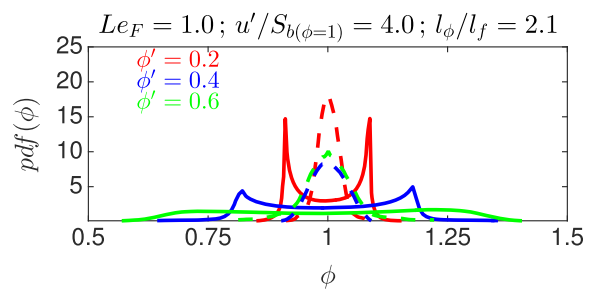

(e)

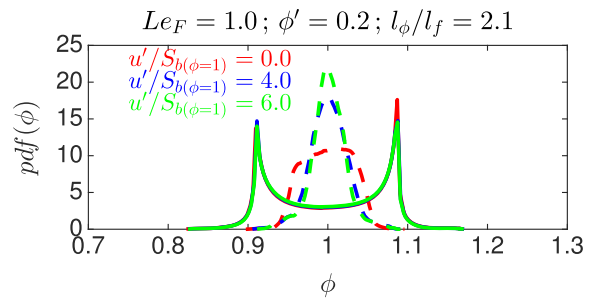

$(\mathrm{g})$

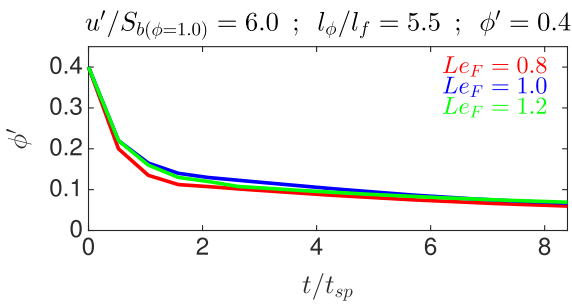

(b)

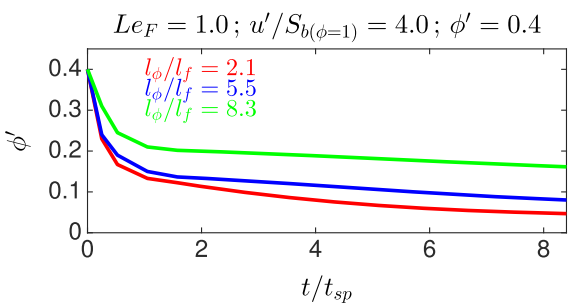

(d)

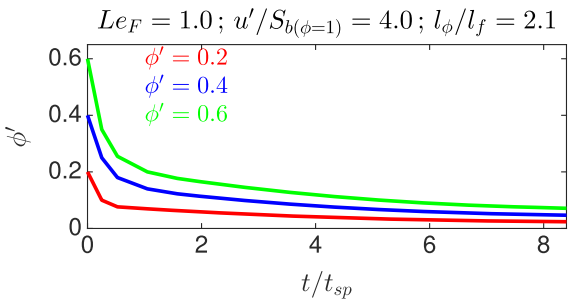

(f)

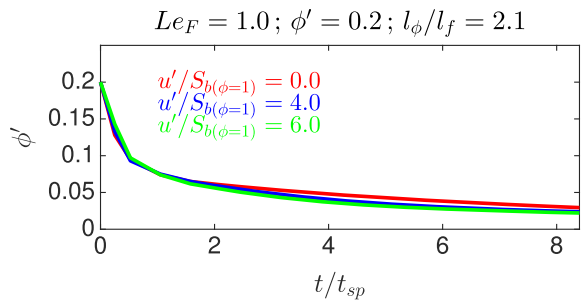

(h)

Fig. 5 (a,c,e,g) Temporal evolution pdf of equivalence ratio for selected cases at $t=1.05 t_{s p}$ [solid line] and $t=8.40 t_{s p}$ [broken line] (first column) and (b,d,f,h) temporal evolution of rms of equivalence ratio $\phi^{\prime}$ evaluated over the whole domain for selected cases (second column)

into the reaction zone at a faster rate than the rate at which heat is conducted out. This leads to simultaneous presence of high temperature and reactant concentration, which increases the probability of finding high values of $\dot{\Omega}_{F}$ in the $L e_{F}=0.8$ case in comparison to that in the $L e_{F}=1.0$ case. Just the opposite mechanism give rise to smaller values of $\dot{\Omega}_{F}$ in the $L e_{F}=1.2$ case in comparison to that in the unity fuel Lewis number case.

The aforementioned effects of $L e_{F}$ on $\dot{\Omega}_{F}$ are in good agreement with previous findings by Chakraborty et al. [10]. Figure 6 ( $3^{\text {rd }}$ row) demonstrates that the fuel reaction rate magnitude distribution remains qualitatively similar following successful ignition and the same 

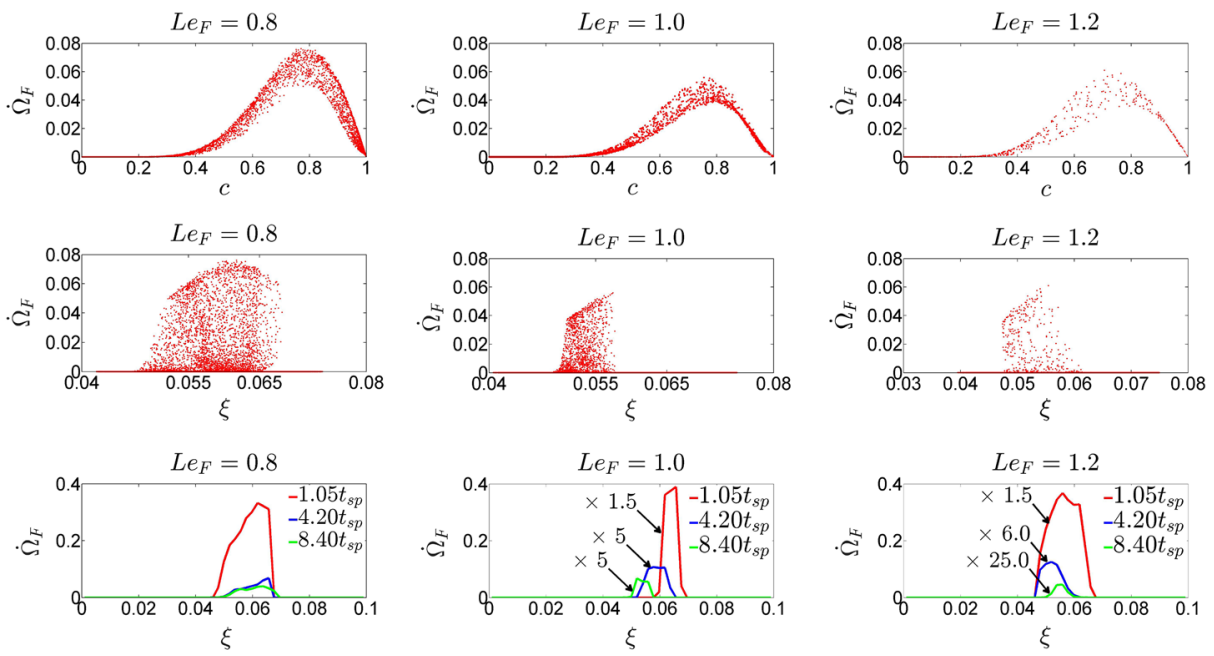

Fig. 6 Scatter of the normalised fuel reaction rate magnitude $\dot{\Omega}_{F}=\left|\dot{w}_{F}\right| \times l_{f} / \rho_{0} S_{b(\phi=1)}$ with reaction progress variable $c\left(1^{\text {st }}\right.$ row $)$, scatter of $\dot{\Omega}_{F}$ with mixture fraction $\xi$ (2nd row), variation of the mean values of $\dot{\Omega}_{F}$ conditional on mixture fraction $\xi$ for reaction progress variable range $0.1 \leq c \leq 0.99$ ( $3^{\text {rd }}$ row). All variation are shown for the case $\phi^{\prime}=0.4 ; l_{\phi} / l_{f}=8.3 ; u^{\prime} / S_{b(\phi=1)}=4.0$ with $L e_{F}=0.8$ ( $1^{\text {st }}$ column), $L e_{F}=1\left(2^{\text {nd }}\right.$ column $)$ and $L e_{F}=1.2\left(3^{\text {rd }}\right.$ column $)$

qualitative behaviour has been observed for other cases. A similar behaviour is observed for all other cases, which is also consistent with previous findings $[8-11,18]$ in the context of localised forced ignition.

\subsection{Effects of $u^{\prime}, \phi^{\prime}, l_{\phi}$ and $L e_{F}$ on the extent of burning}

The extent of burning can be characterised by the burned gas mass $m_{b}$ with $c \geq 0.9$ $[8-11,18]$. The temporal evolutions of the mean and standard deviations of burned gas mass normalised by the mass of an unburned gas sphere with a radius equal to $l_{f}$ (i.e. $[4 / 3] \pi \rho_{0} l_{f}^{3}$ ) for cases with initial $\phi^{\prime}=0.2$ and 0.6 are shown in Figs. 7 and 8 respectively. The variation of the mean and standard deviations of $\left[m_{b}(c \geq 0.9)\right] /\left[\{4 / 3\} \pi \rho_{0} l_{f}^{3}\right]$ for the initial $\phi^{\prime}=0.4$ cases is qualitatively similar to that of the initial $\phi^{\prime}=0.6$ cases, and thus are not explicitly shown here for the sake of conciseness. However, the mean values of $\left[m_{b}(c \geq 0.9)\right] /\left[\{4 / 3\} \pi \rho_{0} l_{f}^{3}\right]$ for initial $\phi^{\prime}=0.4$ cases are greater than the corresponding values obtained for initial $\phi^{\prime}=0.6$ cases. By contrast, the standard deviation of $\left[m_{b}(c \geq 0.9)\right] /\left[\{4 / 3\} \pi \rho_{0} l_{f}^{3}\right]$ for the initial $\phi^{\prime}=0.4$ cases is smaller than the corresponding values obtained for the initial $\phi^{\prime}=0.6$ cases.

The temporal evolutions of mean and standard deviations of $\left[m_{b}(c \geq 0.9)\right] /\left[\{4 / 3\} \pi \rho_{0} l_{f}^{3}\right]$ are re-plotted in Fig. 9, in order to compare the burned gas mass for different values of fuel Lewis number $L e_{F}$ for a given set of initial values of $u^{\prime} / S_{b(\phi=1)}, \phi^{\prime}$ and $l_{\phi} / l_{f}$. It is well known that combustion succeeds only for some realisations even when the turbulent flow statistics are kept unaltered [14, 15, 48-50]. Moreover, the variability of burning statistics in the cylinder of IC engines gives rise to cycle-to-cycle variations [17]. Here, all cases have been analysed for four different realisations of the initial distribution of $\phi$ in order to analyse the degree of variability of the extent of burning. 

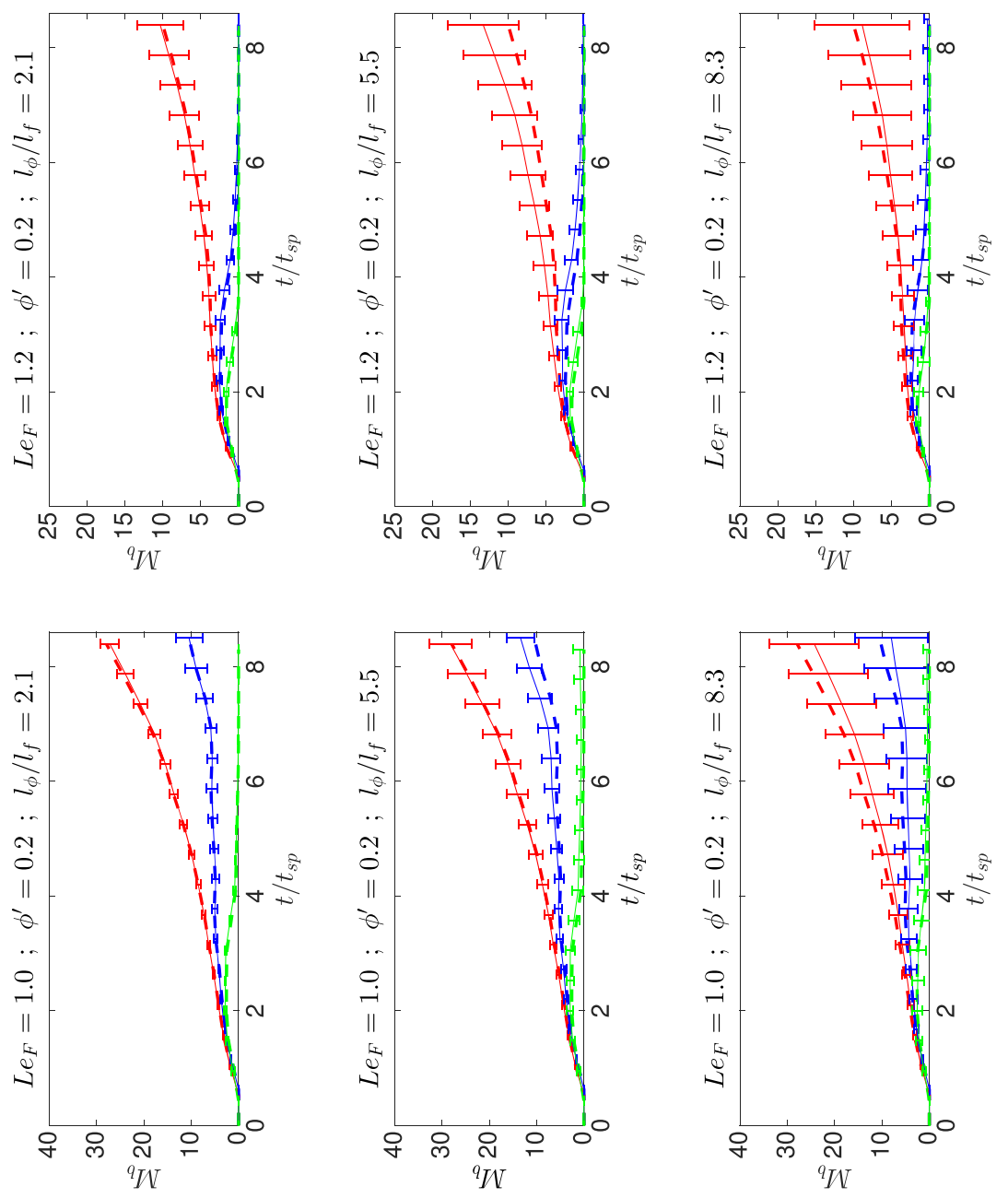

ह
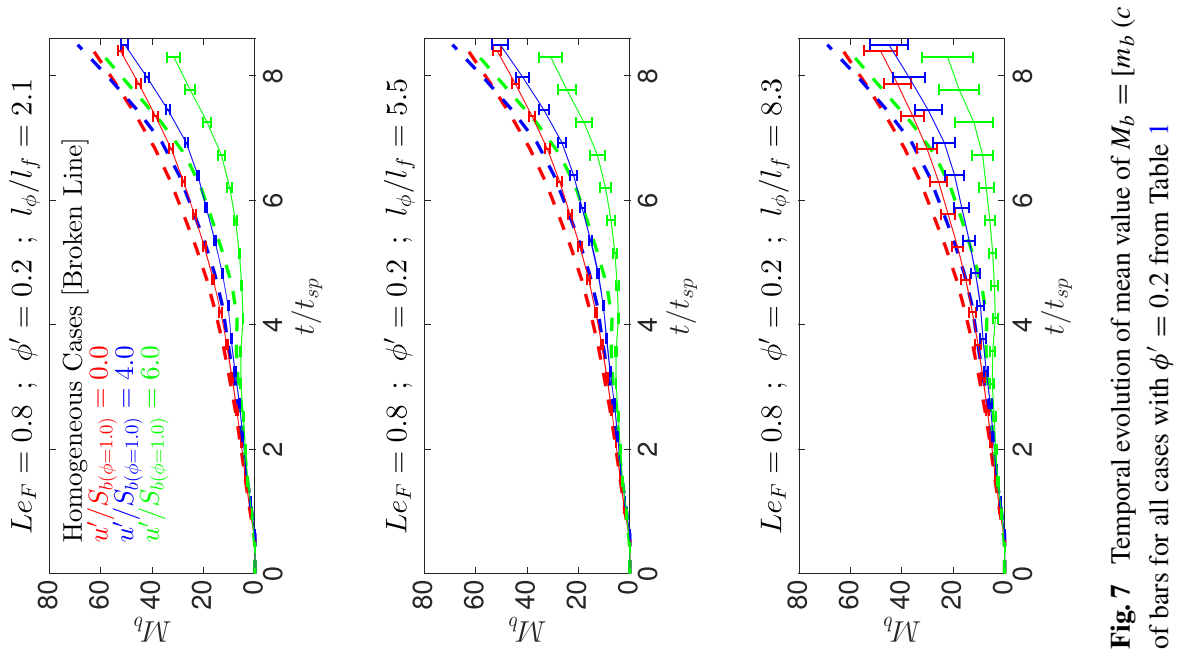

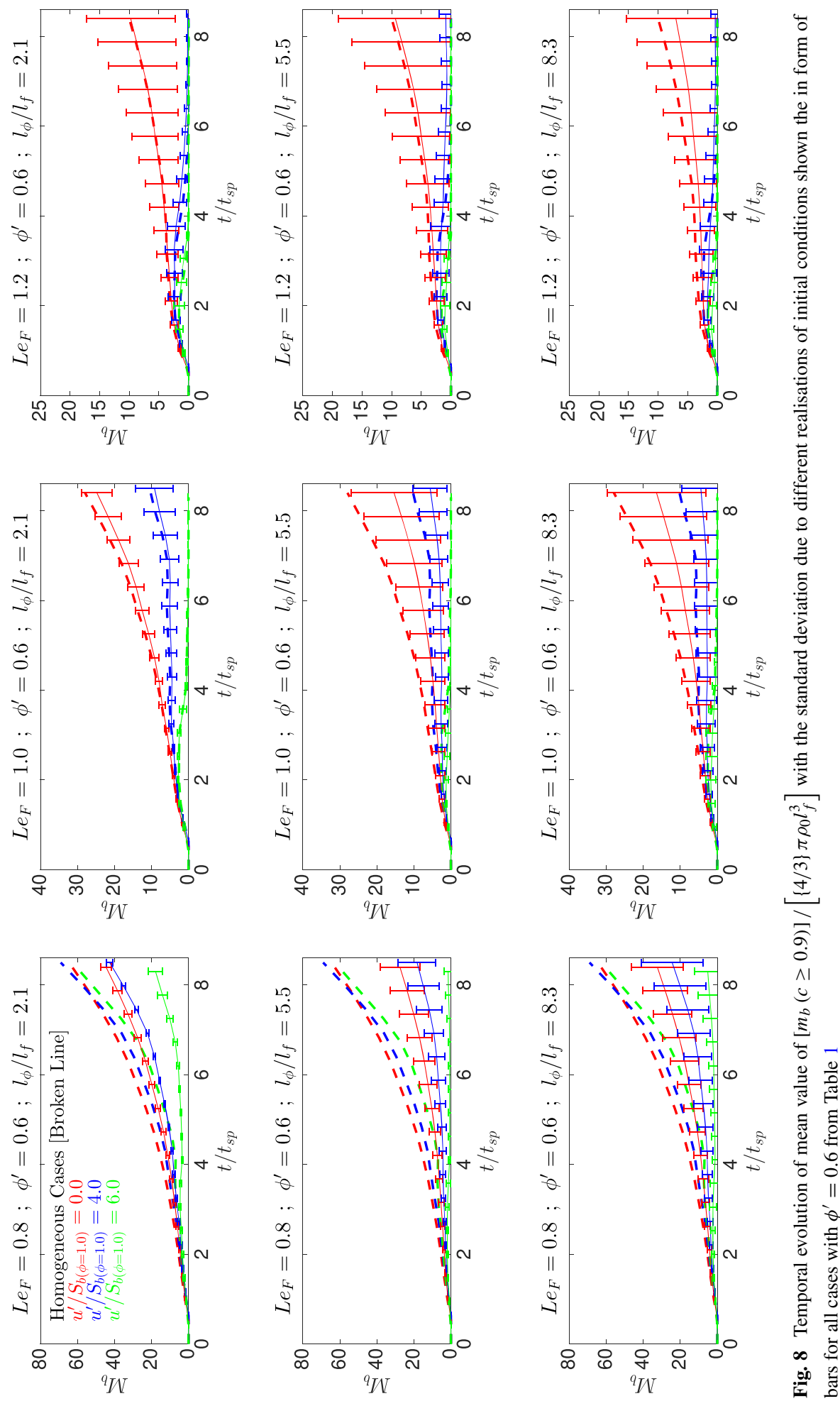

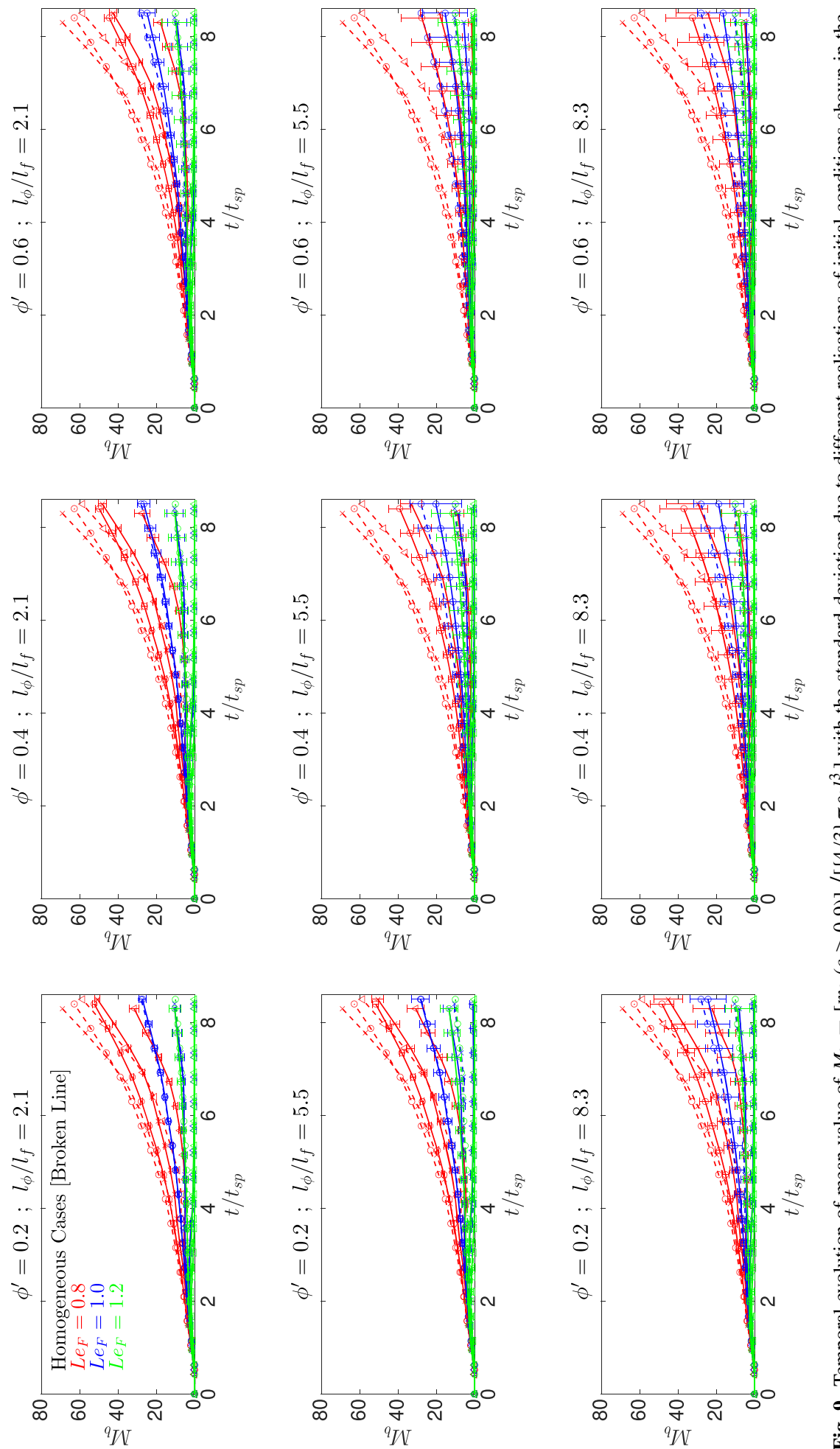

త $\triangleleft$

寒

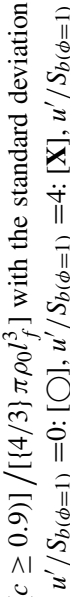

$\underset{\sim}{*}=$

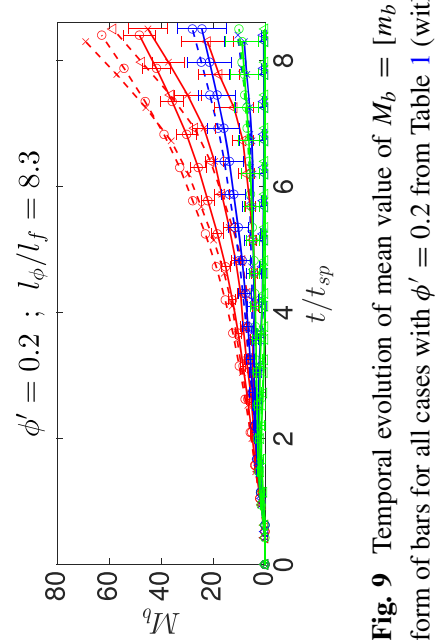


It is evident from Figs. 7-9 that for the extent of burning increases with decreasing $L e_{F}$ for a given set of values of $\phi^{\prime}, l_{\phi} / l_{f}$ and $u^{\prime} / S_{b(\phi=1)}$. The presence of high fuel concentration in the high temperature reaction zone in the $L e_{F}<1.0$ cases (e.g. $L e_{F}=0.8$ ) gives rise to higher extent of burning than the corresponding $L e_{F}=1.0$ cases. By contrast, heat diffuses faster than the rate at which fuel diffuses into the reaction zone in the $L e_{F}>1.0$ cases (e.g. $L e_{F}=1.2$ ) and thus a combination of fuel depletion due to slow mass diffusion rate and low temperature due to high thermal diffusion rate gives rise to smaller extent of burning than the corresponding $L e_{F}=1.0$ cases. It can be seen from Figs. 7-9 that the $L e_{F}=1.2$ cases eventually extinguish for initial values of $u^{\prime} / S_{b(\phi=1)}=4.0$ and 6.0, whereas all the $L e_{F}=0.8$ cases with same initial $u^{\prime}$ exhibit self-sustained combustion. The $L e_{F}=1.0$ cases with initial values of $u^{\prime} / S_{b(\phi=1)}=6.0$ fail to sustain combustion once the ignitor is switched-off for initial values of $\phi^{\prime}=0.4$ and 0.6 irrespective of the values of $l_{\phi} / l_{f}$. Moreover, Figs. 7-9 indicate that an increase in $\phi^{\prime}$ leads to a decrease of $m_{b}(c \geq 0.9)$ for all stratified cases irrespective of $L e_{F}$. The burning rate of mixtures with $\phi<1.0$ and $\phi \geq 1.10$ is smaller than that in the stoichiometric mixtures. The probability of finding $\phi<1.0$ and $\phi \geq 1.10$ increases with increasing $\phi^{\prime}$ and this gives rise to a reduction in burning rate for higher values of $\phi^{\prime}$. The reduction in burning rate due to mixture stratification in globally stoichiometric mixtures is found to be consistent with previous experimental [51] and computational [18, 26, 42, 43, 45] findings.

Moreover, Figs. 7-9 show that an increase in $u^{\prime} / S_{b(\phi=1)}$ leads to a reduction in burning rate for all values of $\phi^{\prime}, l_{\phi} / l_{f}$ for the cases with $L e_{F} \geq 1.0$. An increase in $u^{\prime}$ leads to an increase in eddy diffusivity $D_{t} \sim u^{\prime} L_{11}$ for given value of $L_{11}$, which leads to greater amount of heat loss from the hot gas kernel for high values of $u^{\prime}$. The heat release due to combustion must overcome the heat loss in order to have the growth of the hot gas kernel and self-sustained flame propagation following successful ignition. The probability of findings high values of $c$ decreases with increasing $u^{\prime}$ due to enhanced heat transfer rate from the hot gas kernel for the $L e_{F}=1.0$ and 1.2 cases. This is reflected in the smaller extent of burning for higher values of $u^{\prime}$ for the $L e_{F}=1.0$ and 1.2 cases (e.g. all turbulent cases eventually extinguish for $L e_{F}=1.2$ ). These adverse effect of $u^{\prime}$ on the extent of burning is consistent with previous experimental $[1,5]$ and computational findings [7$11,18]$. The augmented values of flame area generation and burning rate for the turbulent $L e_{F}=0.8$ homogeneous case eclipse the enhanced heat transfer rate from the hot gas kernel, which, in turn, gives rise to higher value of $m_{b}(c \geq 0.9)$ than the corresponding quiescent case.

Furthermore, Figs. 7-9 show that $\left[m_{b}(c \geq 0.9)\right] /\left[\{4 / 3\} \pi \rho_{0} l_{f}^{3}\right]$ remains comparable for the quiescent cases with initial values of $l_{\phi} / l_{f}=5.5$ and $8.3\left(l_{\phi} / l_{f}=2.1,5.5\right.$ and 8.3), and $\phi^{\prime}=0.2$ for the $L e_{F}=1.0$ and $1.2\left(L e_{F}=0.8\right)$ cases. However, $m_{b}(c \geq 0.9)$ in the $L e_{F} \geq 1.0$ cases is found to be smaller for initial values of $l_{\phi} / l_{f}=2.1$ and $\phi^{\prime}=0.2$ than the corresponding cases with initial values of $l_{\phi} / l_{f}=5.5$ and 8.3. Among the turbulent cases with initial $\phi^{\prime}=0.2$, the burned gas mass assumes the highest (lowest) values for the cases with initial $l_{\phi} / l_{f}=5.5\left(l_{\phi} / l_{f}=2.1\right)$ for the $L e_{F}=1.0$ cases, whereas the lowest value of $m_{b}(c \geq 0.9)$ for the $L e_{F}=0.8$ case is obtained for initial $l_{\phi} / l_{f}=5.5$, and the burned gas mass $m_{b}(c \geq 0.9)$ is comparable for initial $l_{\phi} / l_{f}=2.1$ and 8.3 cases. The probability of finding highly reactive mixture corresponding to $1.10>\phi>1.0$ is greater in cases with initial values of $l_{\phi} / l_{f}=5.5$ and 8.3 than in the initial $l_{\phi} / l_{f}=2.1$ cases due to less efficient mixing for initial $\phi^{\prime}=0.2$ with $L e_{F} \geq 1.0$. This gives rise to greater rate of burning in the $L e_{F} \geq 1.0$ cases with initial values of $l_{\phi} / l_{f}=5.5$ and 8.3 than in the initial 
$l_{\phi} / l_{f}=2.1$ cases for initial $\phi^{\prime}=0.2$. However, the aforementioned effect is not strong in the $L e_{F}=0.8$ cases due to higher mixing rate due to greater value of fuel mass diffusivity than in the $L e_{F} \geq 1.0$ cases. The probability of finding slow-burning mixtures $(\phi<1.0$ and $\phi \geq 1.10)$ is greater in the cases with initial $l_{\phi} / l_{f}=5.5$ due to less efficient mixing then that in the initial $l_{\phi} / l_{f}=2.1$ cases, and thus burning rate assumes greater values in the cases with $l_{\phi} / l_{f}=2.1$ than the cases with initial $l_{\phi} / l_{f}=5.5$ when initial $\phi^{\prime}=0.4$ and 0.6 $(0.2,0.4$ and 0.6$)$ for the $L e_{F} \geq 1.0\left(L e_{F}=0.8\right)$ cases. For cases with initial $l_{\phi} / l_{f}=8.3$ the clouds of mixture inhomogeneities are relatively big (see Fig. 1), and as a result, there is a high probability of obtaining a large region of almost homogeneous mixture at the centre of ignitor. If the ignitor centre is located in the vicinity of a large cloud of $1.0 \leq \phi \leq 1.1$, the slow burning rate in the pockets with $1.0<\phi$ and $\phi>1.1$ encountered during the expansion of hot gas kernel is mostly compensated by the high burning rate in the mixture with $1.0 \leq \phi \leq 1.1$ and this leads to greater burned gas mass in the cases with initial $l_{\phi} / l_{f}=8.3$ than in the cases with initial $l_{\phi} / l_{f}=2.1$ when the initial $\phi^{\prime}$ is 0.4 and $0.6(0.2$, 0.4 and 0.6$)$ for the $L e_{F} \geq 1.0\left(L e_{F}=0.8\right)$ cases.

It is evident from Fig. 9 that the increase in heat transfer rate from hot gas kernel with an increase in $u^{\prime}$ leads to a decrease in the extent of burning irrespective of the values of $l_{\phi} / l_{f}$ and $\phi^{\prime}$ for the $L e_{F} \geq 1$ cases but the burned gas mass increases with increasing $u^{\prime}$ for the homogeneous mixture cases with $L e_{F}=0.8$ case. The consumption rate of the fuel in premixed flames increases with increasing $u^{\prime}$ due to increased flame surface area as a result of flame wrinkling. This effect is particularly strong in the $L e_{F}=0.8$ cases where the high values of fuel consumption rate are obtained at the locations, which are convex towards the reactants because of the simultaneous focussing of fuel and defocussing of conductive heat flux. In this configuration, ignition creates a spherical kernel and thus the flame shows a high probability of finding surfaces which are convex towards the reactants. The effects of augmented fuel consumption rate dominate over the increased heat transfer rate in the turbulent homogeneous $L e_{F}=0.8$ cases, and thus the burned gas mass increases with increasing $u^{\prime}$ for these cases. Figure 9 reveals that, for a given value of $l_{\phi} / l_{f}$, the burned gas mass decreases with increasing $\phi^{\prime}$, and the influence of $l_{\phi} / l_{f}$ on the extent of burning have been found to be non-monotonic and dependent on $\phi^{\prime}$ and $L e_{F}$.

\subsection{Effects of initial $\phi$ distribution on the extent of burning}

It can be seen from Figs. 7-9 demonstrate that the variation of $m_{b}(c \geq 0.9)$ between different realisations increases with increasing $l_{\phi} / l_{f}$, whereas the effects of $u^{\prime}$ remain qualitatively similar for all the different realisations. For large values of $l_{\phi} / l_{f}\left(\right.$ e.g. $l_{\phi} / l_{f}=8.3$ ), it is possible to obtain large clouds of both highly flammable and weakly flammable (or noncombustible) mixtures at the ignitor location, which leads to a large variation of $m_{b}(c \geq 0.9)$ between different realisations, and this variability strengthens further with increasing $\phi^{\prime}$ (see large standard deviation values for $l_{\phi} / l_{f}=8.3$ in Figs. 7-9). The findings based on Figs. 7-9 suggest that an ignition system which is designed for fuels with $L e_{F}<1$ may not be sufficient for igniting heavier hydrocarbon-air mixtures with $L e_{F}>1$ but the ignition systems designed for fuels with $L e_{F}>1$ can perhaps successfully be used for lighter fuels with $L e_{F}<1$. Furthermore, the conditions (e.g. a combination of high values of $\phi^{\prime}$ and $l_{\phi} / l_{f}$ ) which lead to large variability between different realisations should be avoided while designing industrial ignition systems for turbulent stratified mixtures. 


\section{Conclusions}

The effects of $l_{\phi} / l_{f}, \phi^{\prime}$ and $u^{\prime} / S_{b(\phi=1)}$ on localised forced ignition of globally stoichiometric stratified mixtures have been investigated using three-dimensional DNS simulations for different values of fuel Lewis number $L e_{F}$ ranging from 0.8 to 1.2. The flame resulting from localised forced ignition shows predominantly premixed mode of combustion although some pockets of non-premixed mode of combustion have also been observed for high values of $\phi^{\prime}, l_{\phi} / l_{f}$ and $L e_{F}$. The extent of burning increases with decreasing $L e_{F}$ for a given set of values of $l_{\phi} / l_{f}, \phi^{\prime}$ and $u^{\prime} / S_{b(\phi=1)}$, energy input and energy deposition duration. Simultaneous presence of high fuel concentration and temperature leads to greater magnitude of fuel reaction rate in the $L e_{F}=0.8$ cases than in the corresponding $L e_{F}=1.0$ cases. By contrast, the combination of the depletion of fuel due to slow fuel diffusion and rapid thermal diffusion rate in the reaction zone leads to weaker burning for the $L e_{F}=1.2$ cases than in the corresponding $L e_{F}=1.0$ cases. The mass of the burned gas region decreases with increasing $L e_{F}$ for a given set of values of $l_{\phi} / l_{f}, \phi^{\prime}$ and $u^{\prime} / S_{b(\phi=1)}$, energy input and energy deposition duration. The initial values of $l_{\phi} / l_{f}$ and $\phi^{\prime}$ have been shown to have significant influences on the self-sustained combustion and the extent of burning following successful ignition. For a given value of $l_{\phi} / l_{f}$, an increase in $\phi^{\prime}$ leads to a reduction of burned gas mass for the stratified mixture cases, whereas the influence of $l_{\phi} / l_{f}$ on the extent of burning has been found to be non-monotonic and dependent on $\phi^{\prime}$ and $L e_{F}$. The increase in heat transfer rate from hot gas kernel with an increase in $u^{\prime}$ leads to a decrease in the extent of burning irrespective of the values of $l_{\phi} / l_{f}$ and $\phi^{\prime}$ for the $L e_{F} \geq 1$ cases but the burned gas mass increases with increasing $u^{\prime}$ for the homogeneous mixture with $L e_{F}=0.8$ case. The above findings demonstrate that the favourable conditions in terms of $l_{\phi} / l_{f}, \phi^{\prime}$ and $u^{\prime} / S_{b(\phi=1)}$ for successful ignition and self-sustained combustion in stratified mixtures are dependent on fuel Lewis number $L e_{F}$. Thus, it is perhaps advantageous to have a feedback mechanism in cylinders of DI engines so that the fuel injection characteristics could be modulated to control $l_{\phi} / l_{f}$ and $\phi^{\prime}$ to ensure successful ignition and self-sustained combustion depending on $u^{\prime}$ in the vicinity of the ignitor. Moreover, it is desirable to avoid the combination of $l_{\phi} / l_{f}$ and $\phi^{\prime}$, which gives rise to large variability in the extent of burning between different realisations. The findings of this numerical investigation indicate that an ignition system for $L e_{F}<1$ may not lead to successful ignition for heavier hydrocarbon-air mixtures with $L e_{F}>1$ but the ignition systems designed for fuels with $L e_{F}>1$ can successfully be used for lighter fuels with $L e_{F}<1$. Although the qualitative nature of the present findings are not likely to change in the presence of detailed chemical kinetics, detailed chemistry DNS for higher turbulent Reynolds number will still be necessary to achieve comprehensive physical understanding and accurate quantitative predictions. Furthermore, it is necessary to assess if the observed effects of $L e_{F}$ disappear beyond a range values of fuel Lewis number, which will require a more extensive parametric analysis than carried out in this paper. Some of the aforementioned issues will form the basis of future investigations.

Acknowledgments The authors are grateful to EPSRC and N8/ARCHER for financial and computational support respectively.

Open Access This article is distributed under the terms of the Creative Commons Attribution 4.0 International License (http://creativecommons.org/licenses/by/4.0/), which permits unrestricted use, distribution, and reproduction in any medium, provided you give appropriate credit to the original author(s) and the source, provide a link to the Creative Commons license, and indicate if changes were made. 


\section{Compliance with Ethical Standards}

Conflict of interests The authors declare that they have no conflict of interests.

Research involving Human Participants and/or Animals: Not applicable for this paper.

Informed consent: All the authors approve this submission.

\section{References}

1. Lefebvre, A.H. Gas Turbine Combustion, $2^{\text {nd }}$ edn., pp. 50-57. Taylor \& Francis (1998)

2. Ballal, D.R., Lefebvre, A.: The influence of flow parameters on minimum ignition energy and quenching distance. Proc. Combust. Inst. 15, 1473-1481 (1975)

3. Ballal, D.R., Lefebvre, A.: Ignition and flame quenching of flowing heterogeneous fuel-air mixtures. Combust. Flame 35, 155-168 (1979)

4. Ballal, D.R., Lefebvre, A.: A general model of spark ignition for gaseous and liquid fuel air mixtures. Proc. Combust. Inst. 18, 737-1746 (1980)

5. Huang, C.C., Shy, S.S., Liu, C.C., Yan, Y.Y.: A transition on minimum ignition energy for lean turbulent methane combustion in flamelets and distributed regimes. Proc. Combust. Inst. 31, 1401-1409 (2007)

6. Poinsot, T., Candel, S., Trouvé, A.: Applications of direct numerical simulation to premixed turbulent combustion. Prog. Energy Combust. Sci. 21, 531-576 (1995)

7. Klein, M., Chakraborty, N., Cant, R.S.: Effects of turbulence on self-sustained combustion in premixed flame Kernels: A Direct Numerical Simulation (DNS) study, Flow Turb. Combust., (accepted) (2008)

8. Chakraborty, N., Mastorakos, E., Cant, R.S.: Effects of turbulence on spark ignition in inhomogeneous mixtures: A Direct Numerical Simulation (DNS) study. Combust. Sci. Technol. 179(1-2), 293-317 (2007)

9. Chakraborty, N., Mastorakos, E.: Direct Numerical Simulations of localised forced ignition in turbulent mixing layers: the effects of mixture fraction and its gradient. Flow Turb. Combust. 80, 155-186 (2008)

10. Chakraborty, N., Hesse, H., Mastorakos, E.: Effects on fuel Lewis number on localised forced ignition of turbulent mixing layers. Flow. Turb. Combust. 84, 125-166 (2010)

11. Wandel, A.P., Chakraborty, N., Mastorakos, E.: Direct Numerical Simulations of turbulent flame expansion in fine sprays. Proc. Combust. Inst. 32, 2283-2290 (2009)

12. Wandel, A.: Extinction indicators in turbulent sprays. Proc. Combust. Inst. 34, 1625-1632 (2013)

13. Wandel, A.: Influence on scalar dissipation on flame success in turbulent sprays with spark ignition. Combust. Flame 161, 2679-2600 (2014)

14. Ahmed, S.F., Mastorakos, E.: Spark Ignition of lifted turbulent jet flames. Combust. Flame 146, 215231 (2006)

15. Ahmed, S.F., Balachandran, R., Mastorakos, E.: Measurements of ignition probability in turbulent nonpremixed counterflow flames. Proc. Combust. Inst. 31, 1507-1513 (2007)

16. Swaminathan, N., Grout, R., Mastorakos, E.: Direct simulation of forced ignition in stratified turbulent mixture. In: Proceedings $3^{\text {rd }}$ European Combustion Meeting. Chania, Greece (2007)

17. Pera, C., Chevillard, S., Reveillon, J.: Effect of residual burnt gas heterogeneity on early flame propagation and on cyclic variability in spark-ignited engines. Combust. Flame 160, 1020-1032 (2013)

18. Patel, D., Chakraborty, N.: Localised forced ignition of globally stoichiometric stratified mixtures: A numerical investigation. Combust. Theor. Model. 18(7), 627-651 (2014)

19. Strehlow, R.A.: Fundamentals of Combustion. International Textbook, Scranton (1968)

20. Glassman, I. Combustion, $2^{\text {nd }}$ edn. Academic Press, New York (1987)

21. Sibulkin, M., Siskind, K.S.: Numerical study of initiation of a combustion wave by an ignition kernel. Combust. Flame 69, 49-57 (1987)

22. He, L.: Critical conditions for spherical flame initiation in mixtures with high Lewis numbers. Combust. Theor. Model. 4, 159-172 (2000)

23. Eswaran, V., Pope, S.B.: Direct Numerical Simulations of the turbulent mixing of a passive scalar. Phys. Fluids 31, 506-520 (1988)

24. Hélie, J., Trouvé, A.: Turbulent flame propagation in partially premixed combustion. Proc. Combust. Inst. 27, 891-898 (1998) 
25. Grout, R., Swaminathan, N., Cant, R.S.: Effects of compositional fluctuations on premixed flames. Combust. Theor. Model. 13, 823-852 (2009)

26. Malkeson, S.P., Chakraborty, N.: A-priori Direct Numerical Simulation analysis of algebraic models of variances and scalar dissipation rates for Reynolds Averaged Navier Stokes Simulations for low Damköhler number turbulent partially-premixed combustion. Combust. Sci. Technol. 182, 960-999 (2010)

27. Chen, J.H., Choudhary, A., Supinski, B. de., DeVries, M., Hawkes, E.R., Klasky, S., Liao, W.K., Ma, K.L., Mellor-Crummey, J., Podhorski, N., Sankaran, R., Shende, S., Yoo, C.S.: Direct Numerical Simulations of turbulent combustion using S3D, vol. 2, p. 015001 (2009)

28. Tarrazo, E., Sanchez, A., Liñán, A., Williams, F.A.: A simple one-step chemistry model for partially premixed hydrocarbon combustion. Combust. Flame 147, 32-38 (2006)

29. Poinsot, T., Echekki, T., Mungal, M.: A study of the laminar flame tip and implications for turbulent premixed combustion. Combust. Sci. Technol. 81(1-3), 45-73 (1992)

30. Louch, D.S., Bray, K.N.C.: Vorticity in unsteady premixed flames: Vortex pair-Premixed flame interactions under imposed body forces and various degrees of heat release and laminar flame thickness. Combust. Flame 125, 1279-1309 (2001)

31. Treurniet, T.C., Nieuwstadt, F.T.M., Boersma, B.J.: Direct numerical simulation of homogeneous turbulence in combination with premixed combustion at low Mach number modeled by the G-equation. J. Fluid Mech. 565, 25-62 (2006)

32. Schroll, P., Mastorakos, E., Cant, R.S.: Direct Numerical Simulations of autoignition in turbulent two phase flows. Proc. Combust. Inst. 32, 2275-2282 (2009)

33. Bilger, R.W.: The structure of turbulent nonpremixed flames. Proc. Combust. Inst. 23, 475-488 (1988)

34. Espí, C.V., Liñán, A.: Fast, non-diffusive ignition of a gaseous reacting mixture subject to a point energy source. Combust. Theor. Modell. 5, 485-498 (2001)

35. Espí, C.V., Liñán, A.: Thermal-diffusive ignition and flame initiation by a local energy source. Combust. Theor. Modell. 6, 297-315 (2002)

36. Neophytou, A., Mastorakos, E., Cant, R.S.: DNS of spark ignition and edge flame propagation in turbulent droplet-laden mixing layers. Combust. Flame 157, 1071-1086 (2010)

37. Ballal, D.R., Lefebvre, A.H.: Ignition and flame quenching in flowing gaseous mixtures. Proc. Roy. Soc. Lond. A. 1977, 163-181

38. Patel, D., Chakraborty, N.: Effects of energy deposition characteristics on localised forced ignition of homogeneous mixtures. Int. J. Spray and Combust. Dyn. 7, 151-174 (2015)

39. Jenkins, K.W., Cant, R.S.: DNS of Turbulent Flame Kernels. In: Liu, C., Sakell, L., Beautner, T. (eds.): Proceeding $2^{\text {nd }}$ AFOSR Conference on DNS and LES, pp. 192-202. Kluwer, Norwell (1999)

40. Poinsot, T., Lele, S.K.: Boundary conditions for direct simulation of compressible viscous flows. J. Comp. Phys. 101, 104-129 (1992)

41. Rogallo, R.S.: Numerical experiments in homogeneous turbulence, NASA TM81315. NASA Ames Research Center, California (1981)

42. Haworth, D., Blint, R., Cuenot, B., Poinsot, T.: Numerical simulation of turbulent propane-air combustion with non homogeneous reactants. Combust. Flame 121, 395-417 (2000)

43. Jiménez, C., Cuenot, B., Poinsot, T., Haworth, D.: Direct numerical simulation and modelling for lean stratified propane-air flames. Combust. Flame 128, 1-21 (2002)

44. Yu, R., Bai, X.-S.: Direct Numerical Simulation of lean hydrogen/air auto-ignition in a constant volume enclosure. Combust. Flame 160, 1706-1716 (2010)

45. Malkeson, S.P., Chakraborty, N.: Statistical analysis of displacement speed in turbulent stratified flames: A Direct Numerical Simulation study. Combust. Sci. Technol. 182, 1841-1883 (2010)

46. Yamashita, H., Shimada, M., Takeno, T.: A numerical study on flame stability at a the transition point of jet diffusion flames. Proc. Combust. Inst. 26, 27-34 (1996)

47. Vedula, P., Yeung, P.K., Fox, R.O.: Dynamics of scalar dissipation in isotropic turbulence: A numerical and modelling study. J. Fluid Mech. 433, 29-60 (2001)

48. Birch, A.D., Brown, D.R., Dodson, M.G.: Ignition probabilities in turbulent mixing flows. Proc. Combust. Inst. 18, 1775-1780 (1981)

49. Rashkovoksky, S.A.: Spark ignition in imperfectly mixed reactants. In: Proceedings of $1^{\text {st }}$ Mediterranean Combustion Symposium, pp. 1403-1411, Anatalya, Turkey (1999)

50. Alvani, R.E., Fairweather, M.: Ignition characteristics of turbulent jet flows. Trans. Ichem. E. 80, 917923 (2002)

51. Renou, B., Samson, E., Boukhalfa, A.M.: An experimental study of freely-propagating turbulent propane-air flames in stratified inhomogeneous mixtures. Combust. Sci. Technol. 176 (1867) 